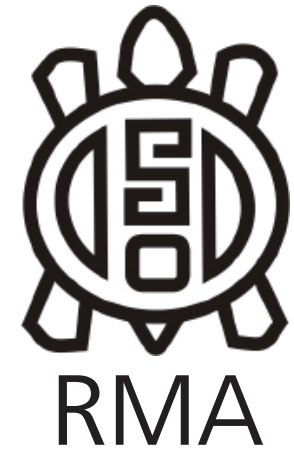

Arqueología

\title{
Estudios biométricos y experimentales de los frutos de Butia odorata y Syagrus romanzoffiana, asociados a la identificación en contextos arqueológicos
}

Biometric and expedrimental studies of Butia odorata and Syagrus romanzoffiana nuts: implications for the archaeological record

*Administración Nacional de Educación Pública, Consejo de Formación en Educación, Uruguay. dsuarez@semipresencial.edu.uy

**Universidad de La República, Centro Universitario Regional del Este-Rocha. Laboratorio de Paleoetnobotánica, Uruguay. Idelpuerto@cure.edu.uy

*** Universidad de La República, Centro Universitario del Regional del Este-Rocha, Laboratorio de Paleoetnobotánica, Uruguay. hinda@cure.edu.uy

\begin{abstract}
Resumen
La presencia de endocarpos carbonizados de Arecaceae nativas, Butia odorata y Syagrus romanzoffiana se ha reportado para diferentes sitios arqueológicos del Este del Uruguay. A nivel de los contextos arqueológicos, la recuperación de endocarpos carbonizados y de instrumentos conocidos como "rompecoquitos" o "piedras con hoyuelos", da cuenta del procesamiento de los frutos de estas especies por parte de poblaciones prehistóricas que habitaron la región. Los frutos de palma, sobre todo de la especie Butia odorata, han sido referidos como uno de los recursos clave para la subsistencia de poblaciones constructoras de montículos ("cerritos de indios") que ocuparon la región a partir del Holoceno medio. No obstante, no existen antecedentes de estudios específicos sobre la identificación taxonómica de los endocarpos de ambas palmas. En este trabajo se aborda la caracterización biométrica del fruto de las especies de Arecaceae a nivel de endocarpos y semillas. También se presentan estudios experimentales referidos a la fracturación mecánica de endocarpos sin carbonizar y flotación de restos naturales y carbonizados. Entre los resultados obtenidos se destacan los valores no solapados de excentricidad de endocarpos de ambas palmas, el comportamiento diferencial de fracturación y flotación y los valores distintivos de masa de endocarpos y semillas de ambas especies. Estos resultados demuestran el potencial de recuperación e identificación taxonómica de endocarpos en contextos arqueológicos de la región.
\end{abstract}

Palabras clave: Arecaceae; Carpología; Biometría; Cerritos de Indios; Paleoetnobotánica.

\begin{abstract}
The presence of native Arecaceae charred nuts has been widely reported in archaeological sites from eastern Uruguay. In such contexts, the recovery of these remains together with stone tools aimed at the obtention of the inner pulp (nutcrackers, locally known as "rompecoquitos" or "piedras con hoyuelos") were interpreted as evidences of palm nuts processing by prehistoric inhabitants of the region. Native palm nuts, particularly from Butia, have been referred as a key subsistence resource for mound builders societies that occupied the area since middle Holocene. Nevertheless, the taxonomic attribution was not supported by any specific study on the matter. In this work we addressed the biometric chracteristics of the fruits of both native Arecaceae species, including seeds and endocarps. Experimental studies were also performed considering the mechanical fracture and flotation of natural and charred remains. Results showed that both palms exhibited distinctive eccentricity values as well as differential flotation and mechanical properties of endocarps and distinctive endocarp and seed mass values. These findings highlighted that palm nut remains from archaeological contexts can be identified with a high degree of confidence.
\end{abstract}

Keywords: Arecaceae; Carpology; Biometrics; Mounds; Paleoethnobotany. 
El sector uruguayo de la Cuenca de la Laguna Merín (CLM) fue ocupado desde hace al menos 8500 años AP, por grupos cazadores-forrajeadores (López, 2013). Las primeras dataciones radiocarbónicas para estas ocupaciones tempranas se obtuvieron a partir de fragmentos de endocarpos carbonizados de frutos de palma (López et al, 2004)

Hacia los 6000-5500 años ${ }^{14} \mathrm{C}$ AP (Holoceno medio) comenzaron a ocupar la región de la CLM grupos de cazadores-forrajeadores de alta eficiencia vinculados a ambientes de alta productividad, como bañados y esteros de gran diversidad biológica (López y Bracco 1994). A partir de estos grupos comenzaría un proceso de aumento poblacional, que implicó un crecimiento en el número de asentamientos y nuevos patrones de ocupación. Se erigen en la geografía del lugar por acción antropogénica, montículos en tierra conocidos localmente como "cerritos de indios". Estos se encuentran asociados a zonas de humedales, aunque también se pueden encontrar en sierras y lomadas (Bracco et al, 2011), pero siempre vinculados a áreas de humedales de alta productividad que concentran recursos (Figura 1). El registro arqueofaunístico, arqueobotánico, también el bioantropológico (como los isótopos estables en huesos humanos, elementos traza y estudios biométricos), indican que los grupos constructores de montículos centraron su economía en la vegetación nativa y las fuentes de origen animal procedentes de los humedales de la CLM (Bracco et al, 2000a; 2008; Bracco, 2006).

Endocarpos carbonizados y fitolitos de hojas de Arecaceae han sido identificados para este periodo en diferentes sitios arqueológicos con cerritos de indios, vinculados
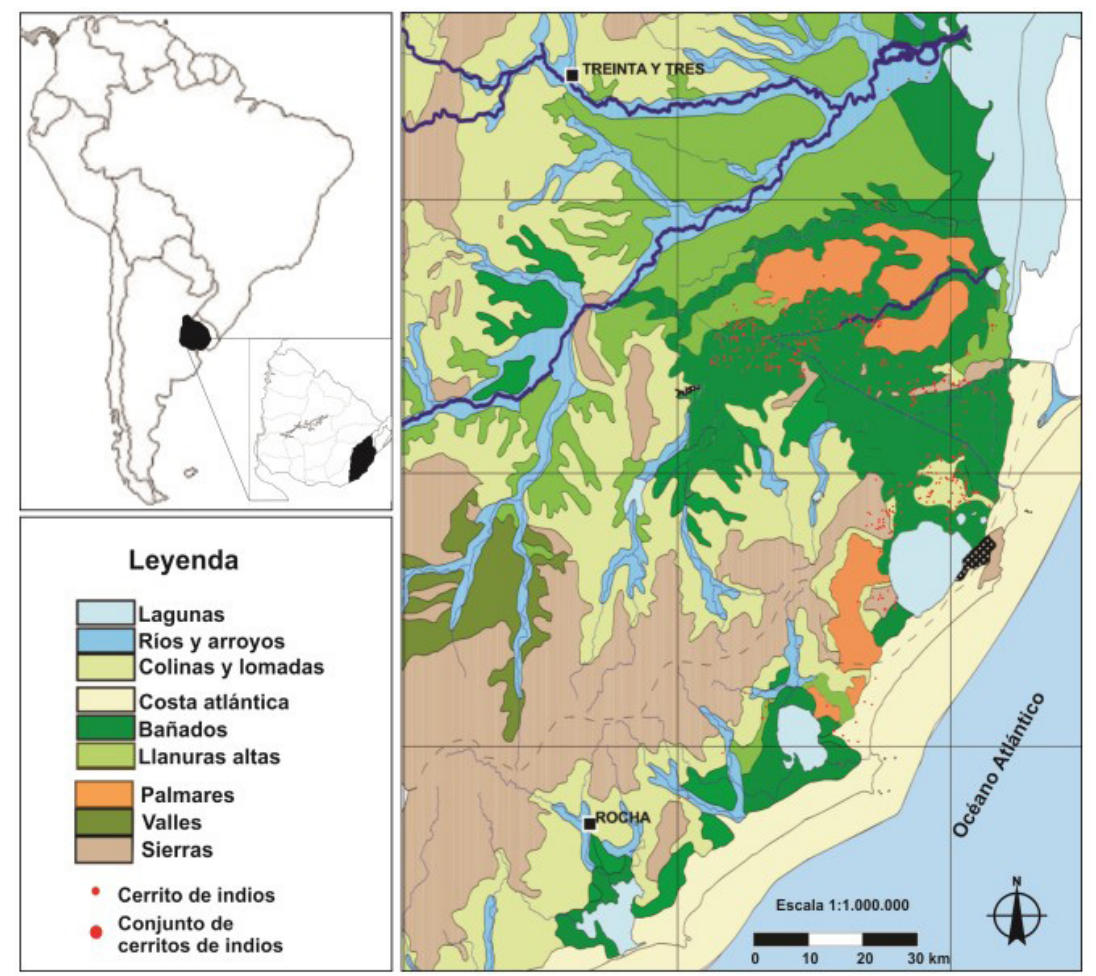

a la CLM (López et al, 2004; del Puerto e Inda, 2008).

Los restos carbonizados de "coquitos" de Arecaceae que datan de este periodo (muchos de ellos con dataciones directas), constituyen un importante indicador del consumo y manipulación de especies de la familia Arecaceae hacia el Holoceno medio. Por otra parte, la presencia de fitolitos de Arecaceae pertenecientes a hojas y frutos en sedimentos provenientes de diferentes sitios arqueológicos (Campos et al, 2001; Iriarte et al, 2001; Capdepont et al, 2002; Iriarte 2003; Capdepont et al, 2005; del Puerto e Inda 2008) testimonia el estatus que tenían estas plantas en la subsistencia de los constructores de cerritos.

El desarrollo tecnológico da cuenta de la especialización en relación al procesamiento del fruto de las palmas, con el hallazgo en sitios de "rompecoquitos", instrumentos líticos a modo de yunque, con una o varias depresiones centrales (López 2001; Bracco et al, 2008), utilizados para fractura de endocarpos. Es importante señalar que no todas las piedras con hoyuelos se vinculan necesariamente o únicamente a estas funciones (Boretto 1970).

La concentración actual de palmeras en el Este del país, en el departamento de Rocha, evidencia la notable presencia de estos recursos naturales en el paisaje. La especie Butia odorata (Barb. Rodr.) Noblick ocupa actualmente alrededor de 70.000 hectáreas (Molina 2001) en esta parte del territorio uruguayo y se halla en franca disminución, por efecto de la ganadería y de la agricultura (Cardoso 1995; Molina 2001; Pezzani 2007). La palmera Syagrus romanzzofiana (Cham.) Glassman, en tanto, integra los bosques serranos y ribereños $y$, aunque rara vez en nuestro país forma palmares, para el departamento de Rocha se ha constatado la formación de pequeños palmares en los alrededores de la Laguna Negra y San Miguel, constituyendo las más densas poblaciones conocidas (Brussa 1998; Brussa et al, 2014).

En base a los registros arqueológicos y descripciones etnográficas, se presume que las palmeras fueron aprovechadas con múltiples propósitos. El uso de hojas de palmeras en cestería, construcciones y entierros, ha sido descripto en el

Figura 1. Distribución geográfica de los cerritos de indios del Este del país.

Figure 1. Geographic distribution of the mounds of the East of the country. 
pasado en grupos indígenas de la Cuenca del Plata (por ejemplo, Serrano 1936; Lozano 1941; Staden 1945, Becker 1976), mientras que el consumo de los frutos también ha sido extensamente descripto (Schiaffino 1925; Ragonese y Martinez-Crovetto 1947; Rodríguez Mattos 1956; entre otros). Junto a los hallazgos arqueológicos y la información etnográfica, la amplia dispersión y gran visibilidad de las palmeras en el paisaje actual llevó a su temprano reconocimiento e integración en las interpretaciones arqueológicas sobre la subsistencia de las poblaciones constructoras de cerritos.

A pesar del reconocimiento que las palmeras tienen en las interpretaciones arqueológicas en Uruguay, no existen trabajos sistemáticos que hayan abordado la identificación taxonómica, caracterización biométrica y aspectos vinculados a la recuperación de endocarpos de contextos arqueológicos. En diferentes trabajos científicos (López y Bracco 1992; Bracco et al, 2000b; López 2001) se hace referencia a la presencia de "coquitos" quemados de las palmeras Butia odorata y Syagrus romanzoffiana, sin acompañamiento de identificación taxonómica; lo cual permite pensar que, si bien esos restos son de palmas, podrían ser de las dos especies o tan solo de una de ellas. Tampoco hay una referencia anatómica de los fragmentos de endocarpos recuperados, siendo que las secciones ecuatoriales por ejemplo tienen dificultad considerable de identificación por especie.

La necesidad de contar con trabajos paleoetnobotánicos con una impronta metodológica vinculada a la identificación de carporestos es de importancia para la arqueología del Este del país y es sin duda una línea en desarrollo.

Si bien existen estudios preexistentes de biometría de endocarpos de Butia odorata y Syagrus romanzoffiana tanto en Uruguay como en Brasil, los mismos no tienen relación con la arqueología (Barilani 2002; de Araujo et al, 2004; Schwartz 2008; Goudel et al, 2013). El antecedente más próximo sobre estudios biométricos de endocarpos vinculados a la arqueología es el trabajo realizado por Bonomo y Capeletti (2014) para el Nordeste argentino en base a las especies Syagrus romanzoffiana y Butia yatay.

En cuanto a estudios de arqueología experimental vinculados a frutos de palma, a nivel nacional se destaca el trabajo de Dabezies y Gazzán (2008) que presenta la relación entre cantidad de frutos ( $\mathrm{gr}$ ) procesados y cantidad de semillas ( $\mathrm{gr}$ ) obtenidas de la actividad extractiva (manual) de semillas de frutos de Butiá en estado inmaduro y maduro. Aunque en el mismo no se analiza la cantidad de fragmentos de endocarpos obtenidos en la fracturación manual, ni se relevan datos biométricos en referencia a los mismos.

A partir de estos antecedentes, el presente trabajo se propuso desarrollar estudios morfométricos y experimentales a partir del fruto de las dos especies nativas del Este de Uruguay: Butia odorata y Syagrus romanzzofiana.

Para llevar a cabo el estudio de los frutos de palma se optó por la siguiente secuencia metodológica: biometría de endocarpos, experimentación (fracturación y flotación), análisis histológicos y bioquímicos.

\section{Objetivo general}

Aportar información al desarrollo de las investigaciones paleoetnobotánicas regionales, en relación a la recuperación, identificación y ponderación de macrorrestos de las palmas Butia odorata y Syagrus romanzzofiana a partir del registro arqueológico.

\section{Objetivos específicos}

Los siguientes objetivos específicos se basan en el análisis comparativo de las especies de palmas: 1) distinguir caracteres diagnósticos, mediante estudios morfológicos y biométricos de endocarpos de ambas especies, que contribuyan a la identificación de restos enteros y fragmentados, provenientes de sitios arqueológicos; 2) mediante fragmentación de endocarpos de ambas especies estimar la equivalencia fragmentos/frutos, para correlacionar con el registro arqueológico; 3) evaluar experimentalmente el potencial de flotación de endocarpos de ambas especies en distintos estados (enteros, fragmentados, carbonizados), para contribuir al ajuste de protocolos de recuperación de macrorrestos vegetales mediante flotación asistida: 4) ponderar el aporte productivo de ambas especies mediante la estimación de masa de semillas, caracterización histológica y bioquímica de los frutos de ambas especies.

\section{Especies analizadas}

A continuación se presentan características ecológicas, botánicas y etnobotánicas de las palmas objeto de estudio.

\section{Butia odorata "Butiá"}

Comunidad vegetal: Pradera, pradera uliginosa, palmar, bosque ribereño y quebrada en serrano.

Forma extensos palmares en el Este del territorio uruguayo, donde ocupa alrededor de 70.000 hectáreas (Molina 2001).

Planta con estípites mayores a $40 \mathrm{~cm}$ de diámetro, cubiertos con restos de pecíolos y de aspecto rugoso (Molina 2001; Brussa y Grela 2007). Con una altura variable del estípite entre 3 a 10 metros (Glassman 1979).

Las hojas pinnaticompuestas, de 2 a $3 \mathrm{~m}$ de longitud curvadas en su extremidad, las pínulas son dísticas y mayores a $1.5 \mathrm{~cm}$ de ancho (Molina 2001; Brussa y Grela 2007). Es destacable la presencia de un pecíolo armado con dientes (Molina 2001). 
Presenta inflorescencias de tipo espádice, protegidas por una bráctea leñosa y glabra llamada espata (Glassman 1979; Molina 2001; Barilani 2002). El espádice porta flores masculinas en su parte distal, flores femeninas y masculinas en la porción central y sólo flores femeninas en la base (Molina 2001); las flores masculinas poseen sépalos y pétalos diferenciados, mientras que las femeninas poseen tépalos (Brussa y Grela 2007).

Fruto drupáceo ovoide de color amarillo a anaranjado. Posee un epicarpo liso y fibroso, menor a $1 \mathrm{~mm}$ de espesor; mesocarpo carnoso, fibroso y amarillo, cerca de $2 \mathrm{~mm}$ de espesor; y endocarpo leñoso, castaño oscuro, cerca de $3 \mathrm{~mm}$ de espesor (Marcato y Pirani 2006).

Las semillas llamadas almendras son de 2 a 3 por fruto, sus formas son ovoides o triangulares (Glassman 1979) y presentan dormancia de seis meses (Mc Currasch 1960). Fenología: florece de setiembre a febrero. Fructifica de diciembre a abril.

\section{Usos actuales}

Alimenticio: La parte más utilizada de la planta es el fruto (butiá) por sus características nutricionales. La pulpa de Butiá es rica en minerales como $\mathrm{Ca}, \mathrm{K}, \mathrm{P}, \mathrm{Mg}, \mathrm{S}, \mathrm{Fe}, \mathrm{Mn}$, $\mathrm{Na}, \mathrm{Al}, \mathrm{Cu}$ y $\mathrm{Zn}$; y es también una fuente de provitamina A y vitamina C (Faria et al, 2008b; Fonseca 2012). Las almendras de $B$. odorata son ricas en lípidos (de 29 a $56 \%$ de contenido por semilla) y en fibras, respecto a los primeros, se destacan el ácido láurico y oleico; también poseen un alto contenido de compuestos fenólicos y carotenoides; en cuanto a los minerales se destacan en mayor proporción potasio, fósforo, magnesio y azufre (Faria et al, 2008a; Sganzeria, 2010; Hoffmann et al, 2014). La almendra también se consume tostada (Cardoso 1995).

Aparte del fruto, la Palma de Butiá permite la extracción de otros productos con valor alimenticio, como lo es la "miel de palma"; a partir de un corte a la altura del cogollo (donde nacen las hojas nuevas) y ahuecándolo con un cuchillo, se drena la savia de esta arecaceae por completo, obteniendo así un líquido dulce que por cocción se espesa y oscurece adquiriendo similitud a una melaza (Cardoso 1995).

Vivienda: uso para construcción y quinchado, así como en la elaboración de cercos vivos (Cardoso 1995).

Industria: Se extrae fibras de las hojas (Cardoso 1995).

Syagrus romanzoffiana "Pindó".

Comunidad vegetal: Bosques ribereños, de quebrada, pantanosos y serrano.

Esta palmera se la encuentra generalmente asociada a montes ribereños, de quebradas y bosquetes pantanosos con una distribución muy amplia que abarca el norte y noroeste del país, forma pequeños palmares (alrededores de la Laguna Negra, San Miguel, etc.) (Brussa et al 2014).

Aunque se adapta a suelos variados, prefiere aquellos de textura franca, profundos, con buen drenaje, ligeramente ácidos (Brussa 1998).

Hojas: pinnadas de 2-4 metros de longitud con las pinnas dispuestas en varios planos a lo largo del raquis, de aspecto desflecado o plumoso (Lorenzi 1992). Tallo: tronco anillado de color grisáceo que puede alcanzar los 12 a 15 m de altura (Lorenzi 1992).

Inflorescencias: ramificadas de hasta 90 centímetros, apareciendo entre las hojas, con flores de color crema protegidas por una espata leñosa (Lorenzi 1992).

Fruto: drupáceo con aproximadamente $2.5 \mathrm{~cm}$ de diámetro, amarillos y presentan pulpa carnosa, fibrosa y endocarpo leñoso. Cada infrutescencia pesa varios kilos cuando los frutos alcanzan la madurez (Brussa y Grela 2007). Fenología: florece intermitentemente, en primavera y verano. Fructifica desde fines de primavera, verano y otoño (Brussa y Grela 2007).

\section{Usos actuales}

Alimenticio: Los frutos son comestibles, constituyen un alimento tradicional de la dieta guaraní (Goudel 2012). Los tallos jóvenes (cogollos) también son consumidos crudos y son un alimento muy valorado (Dujak et al 2015). El azúcar y la fécula contenida en el tronco tumbado comienzan a fermentar, atrayendo gorgojos que depositan sus huevos, estos se transforman en larvas aceitosas, consumidas asadas, fritas cocidas y hasta crudas (Yatsuda 2002).

Implementos domésticos: El racimo de los frutos es utilizado por los guaraníes para escobas de uso doméstico (Yatsuda 2002)

Instrumentos en actividades económicas: Los guayaqui utilizan las fibras de la vaina de la hoja para fabricación de arcos y flechas (Dawson y Gancedo 1977).

Instrumentos musicales: Los guaraníes de Misiones utilizan el estípite para fabricación de tambores. Con las fibras de las vainas foliares se elaboran cuerdas para fijar aros de tambores, cuerdas para violines y cuerdas para guitarras (Keller et al, 2010).

Viviendas: Se utiliza el tronco de la palma para la elaboración de las mismas (Goudel 2012). Se utilizan las hojas para cobertura de construcciones (habitaciones y espacios ceremoniales). También para rompevientos (Yatsuda 2002).

Medicinal: En Brasil su fruto es macerado para obtener un vino o un almíbar, el cual es utilizado como jarabe pectoral (Plotkin y Balick 1983). La raíz posee propiedades 
diuréticas y también es utilizada en el tratamiento de la gonorrea (Darella 1999). Se reportó su uso como anticonceptivo (Toursarkissián 1980).

\section{Metodología}

\section{Biometría}

Identificación de elementos diagnósticos y excentricidad La identificación de frutos provenientes de contextos arqueológicos, implica un estudio detallado con material comparativo (Pearsall 1989). En este sentido, para los frutos de las palmas Butia odorata y Syagrus romanzoffiana, se relevaron caracteres anatómicos tales como espesor del pericarpio, textura y características de la superficie (interna y externa), presencia de suturas, cicatrices de adherencia, naturaleza del exocarpo (grosor, textura y curvatura) y posición de los poros germinativos.

Las dos especies de palmas poseen diferente forma del endocarpo, mientras que en la palmera Pindó es más aovado, la palma Butiá genera frutos con endocarpos globosos.

Para cuantificar lo que la observación revela, se tomaron medidas de los diámetros mayor (D) y ecuatorial (d) de endocarpos $(n=50)$ de ambas palmeras. Los frutos (maduros, del suelo y de la planta) para medición, se colectaron de tres ejemplares diferentes de Butia odorata y Syagrus romanzoffiana del departamento de Rocha.

Para la obtención de los endocarpos se procedió a la extracción mecánica-manual del epicarpo y mesocarpo carnoso. A continuación se midió con calibre el diámetro longitudinal y ecuatorial del endocarpo limpio. Con los datos obtenidos se determinó la variación de la excentricidad para ambas especies. La generación de estos datos es de relevancia para la posterior correlación con los comportamientos de fracturación mecánica de los endocarpos.

Finalmente, se aplicó una Prueba T para muestras independientes con el fin de comparar las medias de los valores obtenidos para cada especie y establecer si existen diferencias estadísticamente significativas. Previo a la aplicación de dicha prueba, se cotejaron los prerrequisitos de normalidad y homogeneidad de varianzas, aplicando los test de Shapiro-Wilk y de Levine respectivamente. Para ello se utilizaron los softwares PAST 3.0 (Hammer y Harper, 2006) e IBM SPSS Statistics 19.

\section{Experimentación}

\section{Fracturación y flotación}

Se procedió a la fragmentación mecánica de 100 endocarpos enteros para cada una de las especies de Arecaceae (Butia odorata y Syagrus romanzoffiana). Para ello se utilizó un "rompecoquitos" de fabricación artesanal, con el que se sometió a los endocarpos a impactos directos con una masa de hierro. El dispositivo consta de dos maderas, una superior y otra inferior de $60 \mathrm{~cm}$ de largo, ésta última con una depresión (en suerficie interna) para posicionar el "coquito" de Arecaceae. Ambas maderas articulan con una bisagra. En el interior del dispositivo se coloca el endocarpo entero, se procede a aproximar las maderas y se aplica un impacto.

Los endocarpos para experimentación se extrajeron de frutos de palmeras provenientes de los departamentos de Canelones (La Paz y Santa Lucía), Montevideo (Museo Jardín Botánico Prof. Atilio Lombardo) y Rocha (Capital).

Los endocarpos de ambas especies fueron procesados en tandas de 10 unidades. Finalizada cada tanda, se procedió a medir la masa en gramos (con balanza de precisión) de los fragmentos de endocarpos obtenidos y de las semillas extraídas.

A partir de los datos obtenidos se calculó la masa promedio por semilla y masa promedio por endocarpo entero. También se contaron los fragmentos obtenidos por fracturación mecánica de endocarpos en cada tanda procesada. Con esos datos se calculó el número promedio de fragmentos de endocarpos generados por unidad (fruto).

Para determinar el potencial de recuperación de fragmentos de endocarpos carbonizados mediante el método de flotación asistida, se diseñó un experimento de flotación. Se tomaron endocarpos de Butia odorata y Syagrus romanzoffiana y se sometieron a diferentes tratamientos: a) fragmentación mecánica de endocarpos enteros y control de flotación; b) fragmentación mecánica de endocarpos enteros, carbonización de los fragmentos y control de flotación; c) endocarpos enteros sometidos a control de flotación y d) endocarpos enteros carbonizados sometidos a control de flotación.

Todos los tratamientos se sometieron a control de flotación en vasos de bohemia de $600 \mathrm{ml}$ con agua destilada y en un tiempo de exposición de quince minutos.

Este diseño experimental tiene como fin determinar el comportamiento de flotación de los endocarpos en diferentes estados (enteros sin quemar, enteros carbonizados, fragmentados sin quemar, fragmentados carbonizados), pues se ha constatado que en los procesos de recuperación arqueológica, los frutos lignificados (drupas o bayas drupáceas) tienen una flotación variable, o incluso hasta nula, en estado de carbonización (Bracco, R., com.pers, febrero 2016).

Mediante registro fotográfico se relevó el resultado del experimento, tras la exposición de los endocarpos en multiestado a control de flotación.

\section{Histología y bioquímica}

Para esta etapa se tomaron endocarpos enteros de las 
especies Butia odorata y Syagrus romanzoffiana y se realizaron cortes transversales de los endocarpos maduros de ambas especies. Los cortes se efectuaron a mano con una hoja de bisturí y se tiñeron con safranina, a partir de la técnica de D'Ambrogio (1986) para materiales leñosos no incluidos. Los cortes se montaron con Entellan ${ }^{\circledR}$. Los preparados obtenidos, se observaron en un microscopio óptico Nikon Eclipse 50i y Premiere MSK-01L BASICO a diferentes aumentos (40x a 50x) para poder analizar los componentes celulares y tejidos vegetales que lo componen.

A nivel de mesocarpo, se extrajo pulpa de los frutos de ambas palmeras y se les aplicó Lugol, para determinación de presencia de granos de almidón (Johansen 1940). La pulpa se extrajo con pinza y bisturí. Una vez agregado el Lugol, se realizaron aplastados de la pulpa con portaobjetos y se llevó la preparación a un microscopio óptico para observación a un aumento de 40x. Se realizó registro fotográfico.

Por último, se realizó un ensayo de detección de lípidos en semillas de ambas especies de palmas. Para esta etapa, se extrajeron semillas de ambos frutos y se realizaron cortes manuales del endosperma con una hoja de bisturí. A las láminas delgadas obtenidas se les aplicó el colorante Sudan III para lípidos (triglicéridos), y se las montó en fresco en portaobjeto para su observación con un microscopio Nikon Eclipse 50i. Se obtuvieron imágenes fotográficas a un aumento de $40 x$, que se procesaron con el software ImageJ ${ }^{\circledR}$.

\section{Resultados}

\section{Morfología y biometría}

A nivel superficial las dos especies presentan tres poros germinativos. La superficie del endocarpo de Butia odorata es lisa con pequeñas depresiones mientras que para Syagrus romanzofiiana los endocarpos a nivel superficial tienen las impresiones de la inserción de fibras que se adhieren al mismo.

Las dos especies poseen diferente forma del endocarpo, mientras que la palmera Pindó es más aovada, la palma Butiá genera frutos con endocarpos globosos. Con los diámetros obtenidos (ecuatorial, longitudinal) de las especies de Arecaceae se obtuvieron datos de excentricidad (Tabla 1) (Figura 2). Los valores obtenidos varían entre 0-1, correspondiendo el primero con un cuerpo totalmente esférico mientras que el segundo refiere a un cuerpo ovoide. Se han observado valores de excentricidad regulares para Syagrus romanzoffiana entre 0,69 y 0,85 , con una media de 0,80 . Mientras que en Butia odorata los valores tienen mayor amplitud y entre 0 y 0,68 tendientes a ser más esféricos y con una media de 0,47.

Los resultados obtenidos con la prueba T para muestras independientes, demuestran que las poblaciones de datos para las tres variables consideradas presentan diferencias estadísticamente significativas entre ambas especies (Tabla 2).

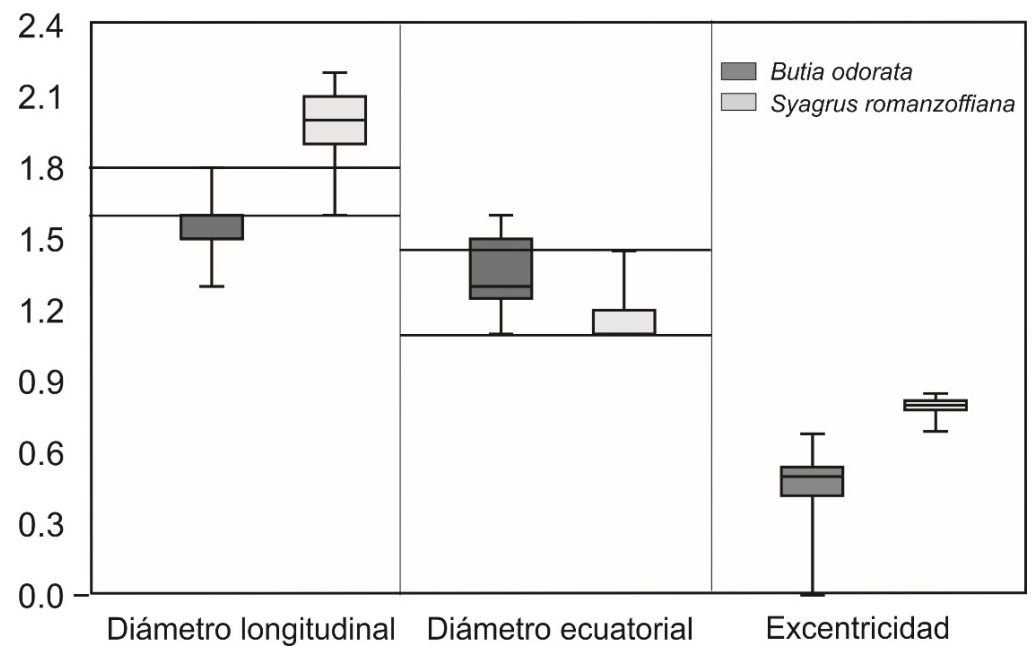

Figura 2. Comparación biometrica de los endocarpos de Butia odorata y Syagrus romanzoffiana

Figure 2. Biometric comparison of the endocarps of Butia odorata and Syagrus romanzoffiana

Tabla 1. Datos biométricos de endocarpos de $B$. odorata $(\mathrm{n}=50)$ S. romanzoffiana $(\mathrm{n}=50)$.

Table 1. Biometric data of endocarps of $B$. odorata $(n=50)$ and S. romanzoffiana $(n=50)$.

\begin{tabular}{|c|c|c|c|c|c|}
\hline \multicolumn{6}{|c|}{ Biometría de endocarpos de Butia odorata y Syagrus romanzoffiana } \\
\hline Especies & Datos relevados & Media & Desvío estándar & Mínimo & Máximo \\
\hline \multirow{3}{*}{ Butia odorata } & $\begin{array}{c}\text { Diámetro } \\
\text { longitudinal } \\
(\mathrm{mm})\end{array}$ & 15,34 & 0,13 & 13,00 & 18,00 \\
\hline & $\begin{array}{c}\text { Diámetro } \\
\text { ecuatorial }(\mathrm{mm})\end{array}$ & 13,38 & 0,12 & 11,00 & 16,00 \\
\hline & Excentricidad & 0,47 & 0,13 & 0 & 0,68 \\
\hline \multirow{3}{*}{$\begin{array}{c}\text { Syagrus } \\
\text { romanzoffiana }\end{array}$} & $\begin{array}{l}\text { Diámetro } \\
\text { longitudinal } \\
(\mathrm{mm})\end{array}$ & 19,84 & 0,13 & 16,00 & 20,20 \\
\hline & $\begin{array}{c}\text { Diámetro } \\
\text { ecuatorial }(\mathrm{mm})\end{array}$ & 11,87 & 0,07 & 11,00 & 14,50 \\
\hline & Excentricidad & 0,80 & 0,04 & 0,69 & 0,85 \\
\hline
\end{tabular}




\begin{tabular}{|c|c|c|c|c|c|c|c|c|}
\hline \multirow{2}{*}{ Variable } & \multirow{2}{*}{\multicolumn{2}{|c|}{ Grupo }} & \multicolumn{3}{|c|}{ Test de normalidad Shapiro-Wilk } & \multicolumn{3}{|c|}{ Test de igualdad de varianza } \\
\hline & & & tatistic & df & Sig. & Varianza & $\mathrm{F}$ & Sig. \\
\hline \multirow{2}{*}{$\begin{array}{l}\text { Diámetro } \\
\text { longitudinal }\end{array}$} & \multicolumn{2}{|c|}{ B. odorata } & ,925 & 50 & ,003 & 0,017086 & \multirow{2}{*}{1,012} & \multirow{2}{*}{0,9666} \\
\hline & & & 909 & 50 & ,001 & 0,016882 & & \\
\hline \multirow{2}{*}{ Diámetro ecuatorial } & B. odorata & & ,923 & 50 & ,003 & 0,015465 & \multirow{2}{*}{2,967} & \multirow{2}{*}{0,0002} \\
\hline & S. romanzoffian & & 867 & 50 & ,000 & 0,0052124 & & \\
\hline \multirow{2}{*}{ Excentricidad } & B. odorata & & 816 & 50 & ,000 & 0,016364 & \multirow{2}{*}{11,295} & \multirow{2}{*}{0,0000} \\
\hline & S. romanzoffian & & ,915 & 50 & ,002 & 0,0014488 & & \\
\hline \multicolumn{9}{|l|}{ Parte B } \\
\hline \multicolumn{9}{|c|}{ t-test para igualdad de medias } \\
\hline \multirow[t]{2}{*}{ Variable } & \multirow{2}{*}{\multicolumn{2}{|c|}{ df }} & \multirow{2}{*}{$\begin{array}{l}\text { Sig. (2- } \\
\text { tailed) }\end{array}$} & \multirow{2}{*}{$\begin{array}{c}\text { Mean } \\
\text { Difference }\end{array}$} & \multirow{2}{*}{$\begin{array}{l}\text { Std. Error } \\
\text { Difference }\end{array}$} & \multicolumn{3}{|c|}{$\begin{array}{c}95 \% \text { Confidence Interval of } \\
\text { the Difference }\end{array}$} \\
\hline & & & & & & Lower & & oper \\
\hline $\begin{array}{l}\text { Diámetro } \\
\text { longitudinal }\end{array}$ & $-17,265$ & 98 &, 0000 &,- 45000 & ,02606 &,- 50172 & &,- 39828 \\
\hline Diámetro ecuatorial & 7,415 & 78,661 & ,0000 & 15080 & ,02034 & 11032 & & 19128 \\
\hline Excentricidad & $-17,579$ & 57,609 & ,0000 &,- 33180 & ,01887 &,- 36959 & &,- 29401 \\
\hline
\end{tabular}

Tabla 2. Las tres variables consideradas presentaron una distribución normal, pero solo el diámetro longitudinal cumplió con el requisito de homogeneidad de varianza entre ambas especies. Debido a ello, la aplicación del test de Student a las variables Diámetro ecuatorial y Excentricidad, se realizó con las modificaciones del test de Welch.

Table 2. The three variables considered presented a normal distribution, but only the longitudinal diameter met the requirement of homogeneity of variance between both species. Due to this, the application of the Student test to the variables Equatorial Diameter and Eccentricity was carried out with the modifications of the Welch test.

\section{Experimentación}

\section{Fracturación}

Se ha determinado que 1 "coquito" de Syagrus romanzoffiana equivale a 14 fragmentos aproximadamente, mientras que 1 de Butia odorata equivale a 21 fragmentos (Figura 3).

En cuanto a la masa de semillas promedio por "coquito", se determinó para Syagrus romanzoffiana una masa promedio de semilla de $0,16 \mathrm{~g}$, mientras que para Butia odorata es de $0,49 \mathrm{~g}$. En lo que respecta a la masa

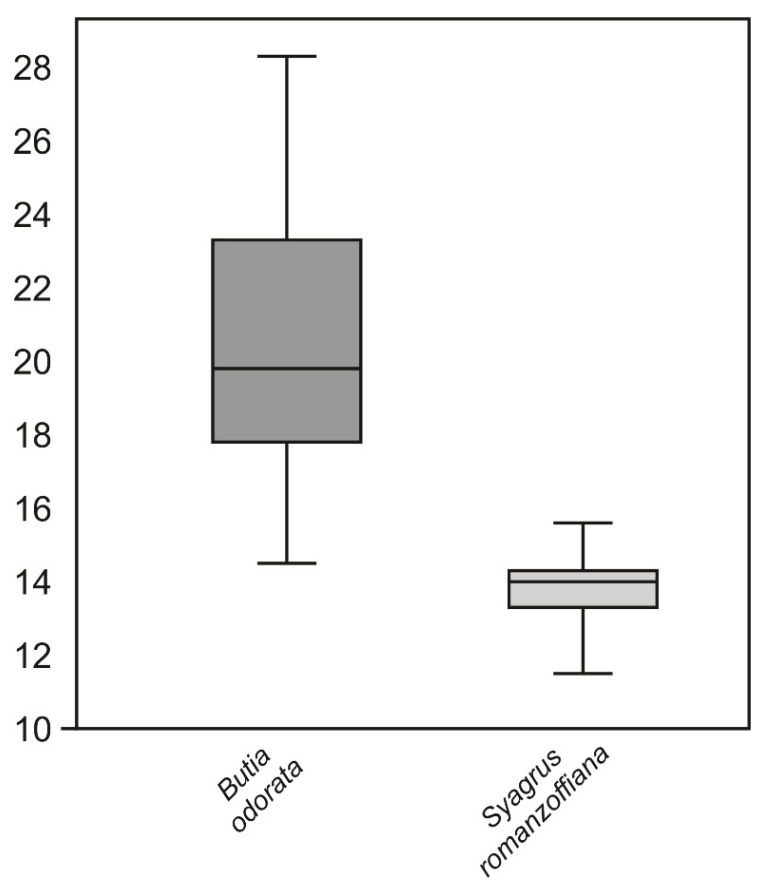

Figura 3. Número de fragmentos por endocarpo fracturado de B. odorata $(n=100)$ S. romanzoffiana $(n=100)$.

Figure 3. Number of fragments per fractured endocarps of $B$. odorata $(n=100)$ and S. romanzoffiana $(n=100)$. promedio de endocarpo por "coquito", para Syagrus romanzoffiana es $1,45 \mathrm{~g}$, siendo para Butia odorata de $1,09 \mathrm{~g}$.

Flotación de frutos de Arecaceae

Mediante experimentación de flotación en el laboratorio, se determinó que para ambas especies de Arecaceae la precipitación de los endocarpos en fase acuosa ocurre cuando éstos están fragmentados, carbonizados o al natural. También se observó que endocarpos enteros sin carbonizar presentan un comportamiento dual, algunos flotan mientras que otros no. En cuanto al estudio de endocarpos enteros carbonizados, para la especie Syagrus romanzoffiana, uno solo de los ejemplares analizados precipitó, el resto se mantuvo a flote, mientras que para la especie Butia odorata, todos los endocarpos enteros precipitaron.

Características histológicas y bioquímicas de los frutos En lo que refiere a características histológicas del endocarpo de ambas especies de palmas, se ha constatado mediante la elaboración de preparados histológicos frescos, que los mismos están formados por esclereidas (células pétreas). En referencia al mesocarpo de Butia odorata, la aplicación de Lugol, a un aplastado del mismo, reveló gran concentración de granos de almidón.

En cuanto a las semillas de ambas especies, el endosperma presenta gran cantidad de cuerpos lipídicos detectados con Sudán III.

\section{Discusión}

\section{Biometría}

Excentricidad

En este trabajo los datos obtenidos del estudio morfométrico de las especies Butia odorata y Syagrus romanzoffiana, brindan información diagnóstica, que permite la diferenciación de ambas especies. 
Con los datos obtenidos de la medición del diámetro longitudinal y ecuatorial, se pudieron establecer valores de excentricidad para cada especie. Los resultados arrojaron valores que no se solapan, lo que indica con claridad, que estas especies desde el punto de vista morfológico, no son confundibles en estado natural.

Si tomamos como referencia el trabajo de Bonomo y Capeletti (2014), con ejemplares de Sygrus romanzoffiana de La Plata (Provincia de Buenos Aires, Argentina), los autores obtuvieron un promedio de diámetro longitudinal $20,43 \mathrm{~mm}$ y ecuatorial de $12,18 \mathrm{~mm}$ con un $\mathrm{n}=100$. Mientras que Goudel et al (2013), a partir de la colecta de ejemplares de Syagrus romanzoffiana de la isla Santa Catarina (Estado de Santa Catarina, Brasil), obtuvieron un promedio de diámetro longitudinal de $19,82 \mathrm{~mm}$ y ecuatorial de $13,73 \mathrm{~mm}$ con un $\mathrm{n}=400$.

En cuanto a los ejemplares procesados de Sygrus romanzoffiana de Rocha en este trabajo, presentan un promedio de diámetro longitudinal de 19,84mm y ecuatorial de $11,87 \mathrm{~mm}$ con $n=50$; estos valores están bastante próximos a lo observado por Bonomo y Capeletti (2014) y Goudel et al (2013).

Para la especie Butia odorata, existen diferentes trabajos de biometría en los cuales se hace medición del diámetro del endocarpo. Sin embargo, los trabajos consultados refieren solo al diámetro ecuatorial. Schwartz (2008) relevó medidas de endocarpos provenientes de tres poblaciones de Santa Vitória do Palmar (Aguiar, Celina y São José), Brasil; los valores promedio de los mismos son $14,07 \mathrm{~mm}$ (zafra 05/06) y 14,18 mm (zafra 06/07) con un $\mathrm{n}=50$. Mientras que De Araujo et al $(2004)^{1}$ obtuvieron valores de diámetro de $14,10 \mathrm{~mm}(\mathrm{n}=300)$, a partir de frutos colectados en el Jardín Botánico de la Universidad Federal de Santa María, Brasil.

En cuanto a los ejemplares procesados de Butia odorata de Rocha, el valor promedio de diámetro ecuatorial es de $13,38 \mathrm{~mm}$ con un $\mathrm{n}=50$. Los valores de las palmeras de Rocha con respecto a los ejemplares brasileros, están por debajo, pero en menos de $1 \mathrm{~mm}$.

\section{Fracturación}

La relevancia de tener datos de fracturación de los "coquitos" de las especies Butia odorata y Syagrus romanzoffiana, está estrictamente vinculada con el registro arqueológico, ya que son estos restos fracturados los que se encuentran con frecuenciaen el procesamiento de los sedimentos. El conocimiento de los procesos de fracturación permite obtener información del comportamiento de los "coquitos" sometidos a acción mecánica y establecer una relación con el registro encontrado.

\footnotetext{
${ }^{1}$ El trabajo no especifica qué diámetro se mide, se asume que es el ecuatorial.
}

Otro de los puntos interesantes para discutir es la correlación entre la excentricidad y el comportamiento de fracturación. Para las dos especies de Arecaceae, se observaron diferentes comportamientos en la fracturación mecánica; cuanto más esférico es el endocarpo mayor es la fragmentación y viceversa, cuanto más elíptico menos fragmenta el endocarpo. A esto hay que sumarle que de las 10 tandas ( $t=10$ frutos) trabajadas para cada especie, solo una correspondiente a Butia odorata, solapó los valores de fragmentación con los de la palmera Syagrus romanzoffiana. Esto se puede explicar por la gran diversidad que existe en los valores de los diámetros entre los frutos de la palma de Butiá.

Si bien este experimento constituye una primera aproximación al comportamiento de fracturación de los edoncarpos de palmas nativas, quedan por hacer registros con diversas variables. Por ejemplo, la fracturación se realizó con un dispositivo de madera, no de piedra como se ha reportado en diversas investigaciones de la arqueología nacional; también quedan por registrar aspectos de la mecánica del golpe como fuerza del impacto (en N [newton]), para esto se debería diseñar otro dispositivo para la fracturación. En cuanto a los frutos, se podría trabajar con endocarpos frescos y otros con un tiempo mayor post-cosecha, para evaluar comportamientos diferenciales de fractura.

Aparte del estudio de fracturación se realizó medición de la masa de los mismos con un $n=100$ frutos. La masa de endocarpo promedio por "coquito" es para Butia odorata de $1,09 \mathrm{~g}$, mientras que para Syagrus romanzoffiana es de 1,45g. Tomando los datos Barilani (2002), la masa promedio de endocarpo que obtuvo de palmeras de Butiá en San Luis ( $n=25)$, fue de 1,5g; mientras que para palmeras de Castillos $(n=25)$ la masa es de $2,2 \mathrm{~g}$. Lo que se puede constatar con estos valores, es la diversidad intraespecífica que existe para las poblaciones de Butiá en el departamento de Rocha. En cuanto a la especie Syagrus romanzoffiana, Goudel et al (2013) determinaron la masa de endocarpo promedio por "coquito" en 2,10g con un $n=400$, a partir de ejemplares de la de isla Santa Catarina, Estado de Santa Catarina, Brasil. Los valores de Goudel et al (2013) están 0,65g por encima de los obtenidos con ejemplares uruguayos; habría que explorar el aumento del número de ejemplares para ver si hay variaciones significativas.

De forma complementaria a la fracturación se realizó medición de masa de las semillas de ambas especies de Arecaceae a partir del procesamiento de 10 tandas de semillas ( $t=10$ unidades). El valor promedio de masa de la semilla para Syagrus romanzoffiana es de 0,16g; los datos de Goudel et al (2013) promedian una masa de $0.23 \mathrm{~g}$ con un mínimo de $0,16 \mathrm{~g}$ y máximo de $0.36 \mathrm{~g}$, nuestros ejemplares están más próximos en peso a los mínimos brasileros. Respecto Butia odorata el valor promedio de masa de la semilla es de 0,49g; los datos de Barilani 
(2002) aportan un promedio de 0,19g, mientras que De Araujo et al (2004) reporta un promedio de 0,30 (frutos con 2 semillas) y 0,25 (frutos con 3 semillas). Los datos obtenidos están bastante por encima de lo reportado por otros autores, por lo que sería necesario realizar una nueva colecta y medición a fin de salvar posibles errores de medición.

En referencia a los datos de masa de semillas y fragmentos de endocarpos obtenidos por fracturación mecánica, se abre todo un campo de discusión con respecto al registro arqueológico y las inferencias teóricas que podemos hacer en torno a la dieta de nuestros pobladores prehistóricos, si tomamos los fragmentos carbonizados como unidad de análisis y ponderación de consumo de frutos de palma.

\section{Características histológicas y bioquímicas de frutos}

En lo que respecta las características histológicas y bioquímicas de los frutos de Butia odorata y Syagrus romanzoffiana, se discuten a continuación los datos obtenidos con trabajos referidos a palmeras de la familia Arecaceae.

Butia odorata, presentó partículas de almidón, en el mesocarpo. Existen diversas referencias bibliográficas sobre la composición de nutrientes del mesocarpo en estas palmeras (Faria et al 2008b, Crosa et al, 2011 y Hoffmann et al, 2014), así como los usos vinculados al consumo de pulpa tanto a nivel prehistórico como actual (Capdepont et al, 2005; Dabezies y Gazzán 2008; Dabezies 2011 y del Puerto 2011).

La constatación de presencia de almidones constituye un paso inicial para la profundización de futuros trabajos a nivel de morfología e integración como otro elemento diagnóstico.

En cuanto al endocarpo, se determinó, que el mismo se compone de esclereidas en ambas palmeras. Básicamente un tipo predominante que son las braquiesclereidas; éstas confieren el carácter esclerosado del endocarpo. Es importante señalar que los datos obtenidos son coincidentes con trabajos a nivel de la familia Arecaceae (Vegas et al, 2008) y géneros Butia y Syagrus (Murray 1973; Reddy y Kulkarni 1985; Manchester et al 2016). Sería de importancia para futuros estudios, ver el comportamiento de estos elementos celulares ante la carbonización, debido a que son células muertas con gruesas paredes lignificadas, de las que no se conoce su estado luego del impacto térmico. Este tipo de trabajos podrían ser útiles para el reconocimiento complementario de endocarpos a nivel microscópico, cuando los fragmentos están próximos al mínimo crítico de análisis (1 $\mathrm{mm})$.

A nivel de las semillas, se detectó la presencia de gotas de lípidos en el endosperma con Sudan III, tanto en Butia odorata como Syagrus romanzoffiana. La presencia de lípidos en el endospermo de otras especies de Arecaceae ya ha sido reportado (Idiem'Opute 1979; de Melo 2017), así como de forma indirecta en la superficie de morteros en trabajos experimentales (Dabezies y Gazzán 2008) en la palma Butiá. El contenido lipídico en semillas de Butiá es de 59.6\% mientras que en Pindó 36.9\% (Idiem'Opute 1979); este contenido graso no debe haber sido para nada despreciable como complemento dietario para los pobladores prehistóricos.

\section{Flotación de Arecaceae}

Mediante experimentación, tomando endocarpos en multiestado, se logró determinar que, tanto en forma natural como en estado de carbonización, los "coquitos" fragmentados no flotan. Este comportamiento no ha sido reportado hasta el momento en trabajos de arqueología nacional y constituye una evidencia significativa para el trabajo con máquina de flotación; ya que de no revisarse los tamices internos de la misma se estaría perdiendo material macrobotánico relevante.

\section{Consideraciones finales}

Los reportes de macrorrestos de frutos de Arecaceae vinculados a sitios arqueológicos (López y Bracco 1992; Bracco et al, 2000b; López 2001) así como de microrrestos (Campos et al 2001, del Puerto e Inda 2001; del Puerto 2015, entre otros), ocupan un lugar importante en las investigaciones arqueológicas del Este de Uruguay. No así los trabajos experimentales referidos a los frutos de palmeras, gravitantes para el análisis de macrorrestos botánicos hallados en contextos arqueológicos.

Dentro de los resultados obtenidos en este trabajo, se destaca la posibilidad de diferenciar, con alto grado de confianza, los endocarpos de ambas especies de palmas analizadas. Esto es válido para los endocarpos completos y en estado fresco, pero también en estado carbonizado y fragmentado.

Este trabajo inicial debería continuarse en un futuro, para estudiar cómo se dan las variaciones de diámetros y excentricidad para frutos en estado de carbonización. Con estos datos se estaría avanzando a una base de datos que sería de gran utilidad para la identificación directa de material arqueológico.

Con respecto al trabajo experimental de fracturación, se da inicio a futuros estudios de correlación entre número de fragmentos y cantidad de frutos procesados, que permitirán no solo obtener datos vinculados a dieta sino también al comportamiento de los restos carbonizados en los sedimentos.

En cuanto a las inferencias que podemos hacer de forma teórica respecto a la relación de fragmentos de endocarpos 
y pulpa o semilla consumidas, destacamos que es significativamente elevada la cantidad de fragmentos que se deben recuperar de los sitios arqueológicos para inferir valores importantes de consumo de frutos de palma.

En referencia a la flotación de endocarpos (multiestado) de ambas palmas, la determinación de un comportamiento dual (flotante y no flotante), hasta ahora no se había reportado para nuestra arqueología y constituye un dato relevante en el ajuste de los protocolos utilizados para la máquina de flotación.

Queda mucho por hacer en futuras investigaciones. Son varios los ajustes experimentales que se pueden hacer en esta línea de investigación: a) aumentar el número de frutos a fragmentar pero utilizando palmeras de diferentes puntos del departamento de Rocha, para salvar el problema de la diversidad; b) trabajar la fragmentación mecánica con "rompecoquitos" exclusivamente, para tener datos más homogéneos (ya que para este trabajo el dispositivo de fractura no era un "rompecoquitos" tradicional); c) estudiar si la carbonización de los fragmentos de Arecaceae aumenta la fracturación; d) investigar la influencia de procesos tafonómicos en la fracturación postcarbonización; e) sistematizar datos que permitan llegar a un modelo predictivo para establecer el número de frutos en estructuras arqueológicas (como los cerritos de indios), a partir de fragmentos de endocarpos.

Rocha, 6 de agosto 2019

\section{Bibliografía}

Barilani, A. (2002). Caracterización de los palmares de Butia capitata (Mart.) Becc. de Castillos y San Luis (Rocha) Incidencia de los coleópteros consumidores de semillas (Doctoral dissertation, Tesis Ing. Agr. Montevideo, Uruguay. Facultad de Agronomía. Universidad de la República).

Becker, Í. I. B. (1976). O índio kaingáng no Rio Grande do Sul. Pesquisas. Série Antropologia Sao Leopoldo, (29), 5-334.

Bonomo, M., y Capeletti, L. E. (2014). Uso prehispánico de las palmeras Syagrus romanzoffiana y Butia yatay en el Nordeste argentino: aportes desde la etnografía y la biometría.

Boretto, R. (1970). Recopilación de antecedentes sobre "Piedras con Hoyuelos" de Argentina, Brasil, Chile, Paraguay y Uruguay. Río Negro: Museo Municipal de Historia Natural de Río Negro.

Bracco, R. (2006). Montículos de la cuenca de la Laguna Merín: tiempo, espacio y sociedad. Latin American Antiquity, 17(4), 511-540.

Bracco, R., Del Puerto, L., Inda, H., Panario, D., Castiñeira,
C., y García-Rodríguez, F. (2011). The relationship between emergence of mound builders in SE Uruguay and climate change inferred from opal phytolith records. Quaternary International, 245(1), 62-73.

Bracco, R.; del Puerto; L., Inda, H. (2008) Prehistoria y Arqueología de la Cuenca de la Laguna Merín. En: Loponte, D.; Acosta, A., (Eds.), Entre la tierra y el agua: arqueología de humedales de Sudamérica, Buenos Aires, Asociación Amigos del Instituto de Antropología y Pensamiento Latinoamericano (AINA), pp.1-59.

Bracco, R., Montaña, J., Bossi, J., Panarello, H., y Ures, C. (2000a). Evolución del humedal y ocupaciones humanas en el sector sur de la cuenca de la Laguna Merín. Arqueología de las Tierras Bajas, 99-116.

Bracco, R; Cabrera, L y López, J. (2000b) La prehistoria de las Tierras Bajas de la Cuenca de la Laguna Merín. En: Bracco, R., Duran, A., (Ed.) Arqueología de las tierras bajas. Montevideo, MEC-Comisión Nacional de Arqueología. pp. 13-38.

Brussa, C. (1998) Conociendo el monte indígena. El Uruguay y sus palmeras. Una familia cimbreante, Montevideo, Suplemento Jardines de El País

Brussa, C. A., y Grela, I. (2007). Flora Arbórea del Uruguay. Con énfasis en las especies de Rivera y Tacuarembó. COFUSA. Mosca. Montevideo, Uruguay, 543.

Brussa, C., Delfino, L., Nicoli, N., Muñóz, F., Gago, J., Rodríguez, R., García, A. (2014). Curso de conocimiento y reconocimiento de flora indígena. IMM

Campos, S., Del Puerto, L., Inda, H., y Castiñeira, C. (2001). Opal phytoliths analysis: its application to the archaeobotanical record in the East of Uruguay. Phytoliths: applications in earth sciences and human history, 129-142.

Capdepont, I., del Puerto, L., y Inda, H. (2005). Instrumentos de molienda: evidencias del procesamiento de recursos vegetales en la laguna de Castillos (Rocha, Uruguay).

Capdepont, I., Del-Puerto, L., e Inda, H. (2002). Caracterización tecnológica y funcional del material cerámico arqueológico de la Cuenca de la Laguna de Castillos. Del mar a los salitrales. Diez mil años de historia Pampeana en el umbral del tercer milenio. Mar del Plata: Universidad Nacional de Mar del Plata-Facultad de Humanidades-Laboratorio de Arqueología-Sociedad Argentina de Antropología.

Cardoso, M. C. (1995). El palmar, la palma y el butiá (No. 582.545 (ARp).

Crosa, M. J., Burzaco, P., Pastorino, N., Irisity, M., Gioscia, D., Ayres, C. (2011) Caracterización fisicoquímica y nutricional 
del fruto Butia capitata y de su pulpa tamizada. En: Innotec (6).

Dabezies, J. M. (2011) Procesando vegetales ayer y hoy: una aproximación a algunos usos actuales de la palma de Butia capitata para entender algunos usos pasados. En: Trama. Revista de Cultura y Patrimonio, 2(3), pp.10-21.

Dabezies, J. M.; Gazzán, N. (2008) Etnoarqueologia en los Tiempos que Corren. En: Actas de las I Jornadas de Jóvenes en Investigación Arqueológica: dialogando con la cultura material, 1, pp.229-236.

D’Ambrogio de Argüeso, A. (1986). Manual de técnicas en histología vegetal, Ana D’Ambrogio de Argüeso.

Darella, M. (1999) Árvores cósmicas/sagradas; o Pindo e o Ygary na cosmovisão dos índios Guarani. Trabalho de pós-gradução, São Paulo, PUC.

Dawson, W.A.; Gancedo, O. A. (1977) La palma pindó (Syagrus romanzoffianum) y su importancia entre los indios guayaqui, En: Obra del centenario del Museo de la Plata, Tomo II (Antropología), pp.339-353

de Araújo Pedron, F., Menezes, J. P., de Menezes, N. L. (2004) Parâmetros biométricos de fruto, endocarpo e semente de butiazeiro. En: Ciência Rural, 34(2), pp.585-586.

de Melo, G. A. M.; de Araújo, M. G. P.; Stauffer, F. (2017). Fruit development and histochemistry of Attalea microcarpa Mart. (Arecaceae-Arecoideae). En: Flora, 235, pp.10-17

del Puerto, L. (2015) Interrelaciones humano-ambientales durante el Holoceno tardío en el Este del Uruguay: cambio climático y dinámica cultural, Tesis de doctorado, PEDECIBA, Montevideo, UdelaR

del Puerto, L. (2011)Ponderación de recursos vegetales silvestres del este del Uruguay: rescatando el conocimiento indígena tradicional. En:Trama. Revista de Cultura y Patrimonio 2(3), pp.22-41.

del Puerto, L., e Inda, H. (2008). Estratégias de Subsistencia y Dinámica Ambiental: Análisis de silicofitolitos en sítios arqueológicos de la cuenca de Laguna de Castillos, Rocha, República Oriental del Uruguay. Fitolitos. Estado actual de sus conocimientos en América del Sur Argentina, 221-236.

del Puerto, L.; Inda, H. (2001) Análisis de silicofitolitos de la matriz sedimentaria del sitio CG14E01, Rocha (Uruguay). En: Natura Neotropicalis 32(2):101-110.

Dujak, M.; Ferrucci, M. S.; Vera Jiménez, M.; Pineda, J.; Chaparro, E.; Brítez, M. (2015) Registros sobre las especies vegetales alimenticias utilizadas por dos comunidades indígenas Mbyá - Guaraní de la Reserva para Parque Nacional San Rafael, Itapúa - Paraguay.
Faria, J.P.; Arellano, D.B.; Grimaldi, R.; Silva, L.C.R.; Vieira, R.F.; Silva, D.B.; Agostini-Costa, T.S. (2008a) Chemical characterization of nut of Butia capitata var. capitata. En: Revista Brasilera Fruticola, 30, pp.549-552.

Faria, J.P., Almeida, F., Silva, L.C.R., Vieira, R.F., AgostiniCosta, T.S. (2008b) Chemical characterization of pulp of Butia capitata var. capitata. En: Revista Brasilera Fruticola, 30, pp.827-829.

Fonseca, L. X. (2012). Caracterização de frutos de butiazeiro (Butia odorata Barb. Rodr.) Noblick y Lorenzi e estabilidade de seus compostos bioativos na elaboração e armazenamento de geleias. Tesis de posgrado del Programa de Pós-Graduação em Ciência e Tecnologia Agroindustrial. Universidad de Pelotas.

Glassman, S. F. (1979). Re-evaluation of the genus Butia with a description of a new species [Butia purpurascens, Brazil]. Principes (USA).

Goudel, F. (2012). Caracterização e processamento de mapuitã, os frutos da palmeira jerivá (syagrus romanzoffiana cham), Dissertação apresentada como requisito parcial ao título de Mestre em Agroecossistemas, Florianópolis, Universidade Federal de Santa Catarina.

Goudel, F.; Shibata, M.; Coelho, C. M. M.; Miller, P. R. M. (2013) Fruit biometry and seed germination of Syagrus romanzoffiana. En: Acta Botanica Brasilica, 27(1), pp.147154.

Hammer, O., Harper, D. A. T., y Ryan, P. D. (2006). Paleontological statistics, version 1.57 .

Hoffmann, J. F., Barbieri, R. L., Rombaldi, C. V., y Chaves, F. C. (2014). Butia spp.(Arecaceae): an overview. Scientia Horticulturae, 179, 122-131.

Idiem'Opute, F. (1979) The seed lipids of the palm family. En: Journal of the American Oil Chemists' Society, 56(4), pp.528-530.

Iriarte, J. (2003). Mid-Holocene emergent complexity and landscape transformation: the social construction of early formative communities in Uruguay, La Plata Basin.

Iriarte, J., Holst, I., Lopez, J., y Cabrera, L. (2001). Subtropical wetland adaptations in Uruguay during the Mid-Holocene: an archaeobotanical perspective.

Johansen, D. A. (1940). Plant microtechique. McGraw-Hill Book Company, Inc.; London

Keller, H. (2010) La presencia en Argentina de Berberis laurina Billb. (Berberidaceae), una especie de uso múltiple. En: Kurtziana, 35 (1), Córdoba, Instituto de Botánica del Nordeste, UNNE-CONICET. 
López, J. M. (2001). Las estructuras tumulares (cerritos) del Litoral Atlántico uruguayo. Latin American Antiquity, 12(3), 231-255.

López, J. M., A. Gascue y F. Moreno (2004) La Prehistoria del Este de Uruguay: Cambio Cultural y Aspectos Ambientales. Anales de Prehistoria y Arqueología 20:9-24.

López, J. y Bracco, R. (1994). Cazadores-recolectores de la Cuenca de la Laguna Merín: aproximaciones teóricas y modelos arqueológicos. Arqueología Contemporánea, $5,51-64$.

López, J. y M, Bracco, R. (1992) Relación Hombre-Medio Ambiente en las poblaciones prehistóricas de la Zona Este del Uruguay. En: Troncoso y Van der Hammen (eds) Archaeology and Environment in Latin America. Amsterdam, pp.259-282.

López, J.M. (2013) La pre historia y proto-historia de las tierras bajas del este de Uruguay y sur de Brasil. Prehistoria de Rocha: apuntes sobre poblamiento temprano de estas tierras. Ministerio de Educación y Cultura, Comuna de Rocha.

Lorenzi, H. (1992). Arvores Brasileiras. Sao Paulo, Editorial Plantarum Ltda. Pp.172-196.

Lozano, P. (1941). Descripción corográfica del terreno, ríos, árboles y animales de las dilatadísimas provincias del Gran Chaco, y de los ritos y costumbres de las innumerables naciones bárbaras e infieles que la habitan. Con una cabal relación histórica de lo que en ellas han obrado para conquistarlas algunos gobernadores, ministros reales y misioneros jesuitas para reducirlas a la fe del verdadero Dios [1773], reedición en Tucumán, Instituto de Antropología.

Manchester, S. R., Bonde, S. D., Nipunage, D. S., Srivatava, R., Mehrotra, R. C., Smith, S. Y. (2016). Trilocular Palm Fruits from the Deccan Intertrappean Beds of India. En: International Journal of Plant Sciences, 177(7), pp.633-641.

Marcato, A. C., y Pirani, J. R. (2006). Flora de Grão-Mogol, Minas Gerais: Palmae (Arecaceae). Boletim de Botânica, 24(1), 1-8.

Mc Currach, J. C. (1960). Palms of the world. New York, Harper and Brothers Ed.

Molina, B. (2001). Biología y conservación del palmar de butiá (Butia capitata) en la Reserva de Biosfera Bañados del Este. Avances de investigación. PROBIDES. Rocha, Uruguay. Documento de trabajo, 34, 33.

Murray, S. G. (1973). The formation of endocarp in palm fruits. In: Principes, 17(3), pp.91-102.
Pearsall, D. (1989) Techniques for Recovering Macroremains. Paleoethnobotany, A Handbook of Procedures. San Diego, EstadosUnidos, Ed, Academic Press, Inc.

Pezzani, F. (2007). Los palmares de Butia capitata en la Reserva Bañados del Este: Un escenario para la conservación y el desarrollo, Uruguay. Reservas de la Biosfera, 201.

Plotkin M. J., Balick M. J. (1983) Medicinal Uses of South American Palms. In: Journal of ethnopharmacoloqy. 10(2), pp.157-179.

Ragonese, A. E., y Crovetto, R. M. (1947). Plantas indígenas de la Argentina con frutos o semillas comestibles. Revista de Investigaciones Agrícolas, 1(3), 147-216.

Reddy, G. N.; Kulkarni, A. R. (1985) Contribution to the anatomy of palm fruits-Cocosoid palms. In: Proceedings: Plant Sciences, 95(3), pp.153-165

Rodríguez Mattos, J. (1956) Estudios pomológicos de frutos indígenas de Rio Grande do Sul, fasc. 2, Porto Alegre, Ed. Santana.

Schiaffino, R. (1925) Historia de la medicina en el Uruguay, (Tomo I), Montevideo, Ministerio de Salud Pública.

Schwartz, E. (2008). Produção, fenologia e qualidade dos frutos de Butia capitata em populações de Santa Vitória do Palmar.

Serrano, A. (1936). Etnografía de la antigua provincia del Uruguay.

Sganzeria, M. (2010). Caracterização físico-química e capacidade antioxidante do butiá (Master's thesis, Universidade Federal de Pelotas).

Staden, H. (1945). Viajes y cautiverio entre los caníbales. Editorial Nova.

Toursarkissián, M. (1980) Plantas Medicinales de la Argentina. Buenos Aire, Ed. Hemisferio Sur.

Vegas, C., Millán, B., Pintaud, J.CH., Kahn, F. (2008) Anatomía del fruto y perianto en especies peruanas del género Astrocaryum (Arecaceae): descripción e importancia taxonómica, En: Revista peruana de biología: las palmeras en América del Sur 15(1), pp.085- 096

Yatsuda, A. (2002) Práticas fitotécnicas de uma comunidade indígena Mbya Guarani, Varzinha, RS: da roça ao artesanato. Tese (Doutorado em Fitotecnia), Faculdade de Agronomia, Universidade Federal do Rio Grande do Sul. Porto Alegre. 